\title{
Cryptococcal Pneumonia
}

National Cancer Institute

\section{Source}

National Cancer Institute. Cryptococcal Pneumonia. NCI Thesaurus. Code C35361.

Fungal pneumonia caused by inhalation of particles of Cryptococcus. It usually occurs in immunocompromised patients such as persons with AIDS, transplant recipients, patients receiving cytotoxic chemotherapy, and patients with hematologic malignancies. It is rare in immunocompetent individuals. Signs and symptoms include fever, cough, and dyspnea. 